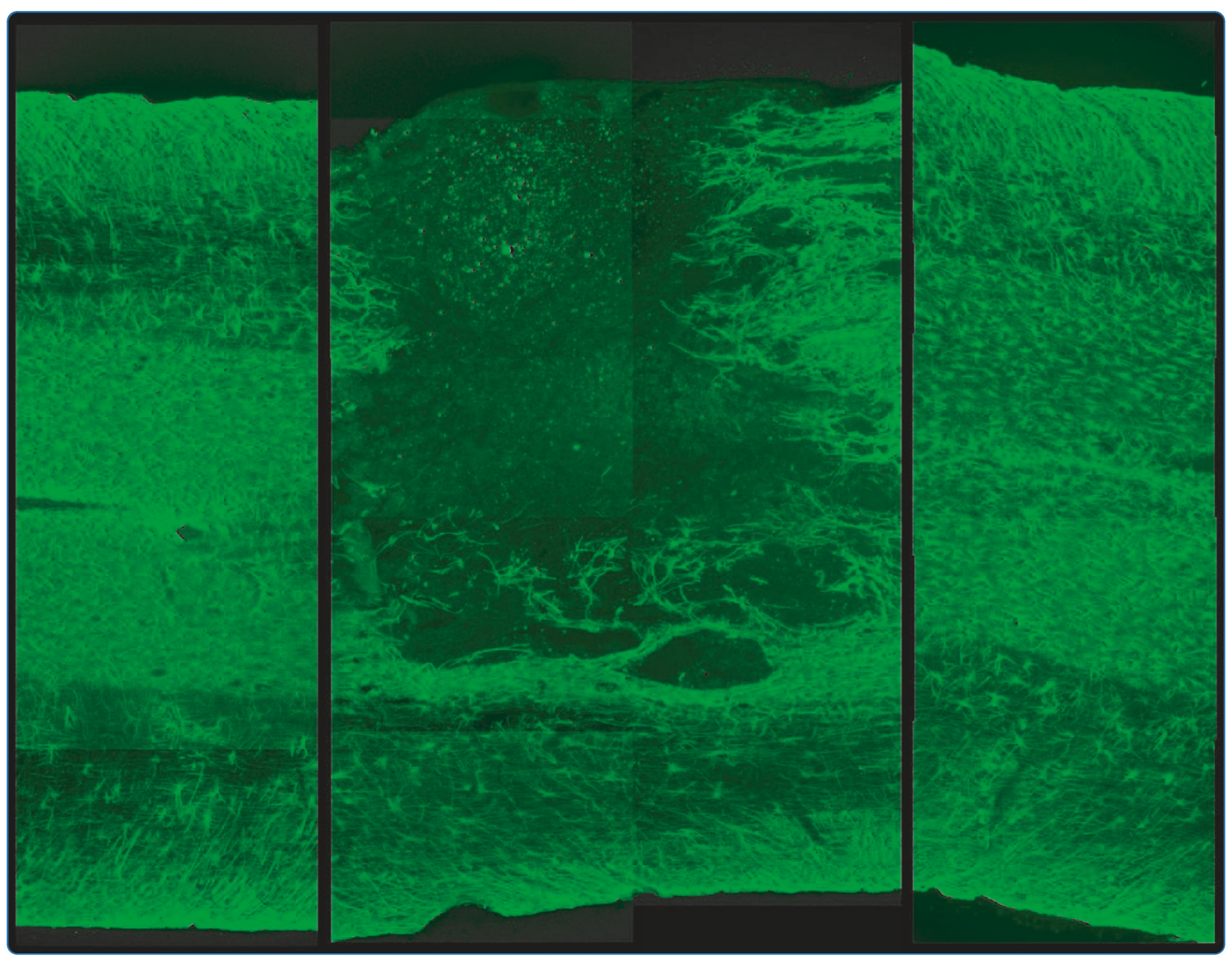

Macrophage presence is essential for the regeneration of ascending afferent fibres following a conditioning sciatic nerve lesion in adult rats

Aguilar Salegio et al. 


\title{
Macrophage presence is essential for the regeneration of ascending afferent fibres following a conditioning sciatic nerve lesion in adult rats
}

Ernesto A Aguilar Salegio, Anthony N Pollard, Malcolm Smith, Xin-Fu Zhou*

\begin{abstract}
Background: Injury to the peripheral branch of dorsal root ganglia (DRG) neurons prior to injury to the central nervous system (CNS) DRG branch results in the regeneration of the central branch. The exact mechanism mediating this regenerative trigger is not fully understood. It has been proposed that following peripheral injury, the intraganglionic inflammatory response by macrophage cells plays an important role in the pre-conditioning of injured CNS neurons to regenerate. In this study, we investigated whether the presence of macrophage cells is crucial for this type of regeneration to occur. We used a clodronate liposome technique to selectively and temporarily deplete these cells during the conditioning phase of DRG neurons.

Results: Retrograde and anterograde tracing results indicated that in macrophage-depleted animals, the regenerative trigger characteristic of pre-conditioned DRG neurons was abolished as compared to injury matchedcontrol animals. In addition, depletion of macrophage cells led to: (i) a reduction in macrophage infiltration into the CNS compartment even after cellular repopulation, (ii) astrocyte up-regulation at rostral regions and downregulation in brain derived neurotrophic factor (BDNF) concentration in the serum.
\end{abstract}

Conclusion: Activation of macrophage cells in response to the peripheral nerve injury is essential for the enhanced regeneration of ascending sensory neurons.

\section{Background}

Injured axons in the mammalian central nervous system (CNS), compared to those in the peripheral nervous system (PNS), do not regenerate. The limited capacity of matured CNS axons to regenerate has been attributed to the presence of an inhibitory barrier formed by myelin and its associated molecules [1], especially when expressed after injury $[2,3]$. Consequently, many of the damaged axons undergo atrophy and form 'dystrophic end balls' indicative of halted attempts at regeneration [4]. The use of peripheral nerve grafts to circumvent inhibitory cues in the injured CNS environment have been successful at stimulating axonal regeneration $[1,5,6]$ and have provided important evidence for the

\footnotetext{
* Correspondence: xin-fu.zhou@flinders.edu.au

Department of Human Physiology and Centre for Neuroscience, Flinders University, GPO Box 2100, Adelaide 5001, Australia
}

intrinsic ability of adult injured neurons to regrow and remain in a regenerative mode within the CNS.

In dorsal root ganglion (DRG) neurons, injury to the peripheral branch in the form of a sciatic nerve injury (SNI) prior to injury to the CNS branch (spinal cord - dorsal column cut), has been previously shown to result in axonal regeneration of centrally projecting ascending fibres [7]. This is referred to as conditioning of DRG neurons and it demonstrates the regenerative capacity of injured fibres in the matured CNS. However, the exact mechanism mediating this regenerative trigger is not fully understood. It has been proposed that activation of macrophage cells, together with satellite cell activation and proliferation within the DRG, as part of the normal PNS response to injury, might be playing a crucial role [8].

Interestingly, the exogenous application of cAMP (cyclic adenosine monophosphate) to DRGs in vivo can mimic the regeneration of the injured CNS branch, 
without injury to the peripheral DRG branch [9]. Mechanistically, this regenerative response after cAMP administration, has been attributed to the blocking and/ or reduction in sensitivity of axons to myelin inhibitors in the CNS [10]. Elevation in cAMP by priming and/or exposing neurons in vitro to trophic factors such as brain derived neurotrophic factor (BDNF) resulted in neurite outgrowth in the presence of inhibitory molecules such as myelin and myelin-associated glycoprotein (MAG) [11]. This is important given that endogenous neurotrophic factors such as BDNF and cilliary neurotrophic factor (CNTF) have been shown to be essential for enhancing regeneration [12-15].

Recently, we and others have demonstrated that after SNI, there is a robust macrophage response infiltrating DRGs [16] and even other parts of CNS such as the spinal cord [17] and optic nerve [18]. In addition, further evidence from our laboratory indicates that macrophages can express a number of neurotrophic factors such as BDNF, NGF, and other neurotrophic cytokines [19]. In this study, we hypothesized that macrophage cells play a crucial role in the conditioning and regeneration of the CNS-DRG branch (i.e. afferent fibres). To test this, we selectively and temporarily depleted macrophage cells during the conditioning phase of DRG neurons (i.e. prior to, during and after $\mathrm{SNI}$ ) via the intravenous delivery of a liposome-encapsulated clodronate, a technique described to result in macrophage suicide $[20,21]$. We found that the temporal depletion of these cells completely abolished the regenerative trigger characteristic of this model and therefore, we propose a beneficial role of macrophage cells in the regeneration of pre-conditioned DRG neurons.

\section{Methods}

\section{Animals}

Adult female Sprague Dawley (SD) rats (10-12 weeks) were used under the guidelines of the National Health and Medical Research Council of Australia and approved by the Animal Welfare Committee of Flinders University of South Australia.

\section{Treatment Groups}

Animals were divided into 2 experimental groups, both of which received a SNI (day 0 ) followed by a dorsal column cut (DCC, day 7). The timing of the CNS injury relates to the optimal conditioning of DRG neurons, known to occur seven days after SNI, resulting in the maximum amount of CNS regeneration possible [7]. Control animals $(n=10)$ received intravenous (iv) tail vein injections of sterile saline alone $(2 \mathrm{ml} /$ injection). Test animals $(\mathrm{n}=10)$ received iv tail vein injections of liposome-encapsulated clodronate ( $2 \mathrm{ml} /$ injection). In both of these two groups sterile saline was administered as the vehicle during iv delivery and the only difference between them was the use of liposomes in the treatment group. Note that control animals did not receive liposome-encapsulating saline given the possibility that liposomes alone may "block macrophage phagocytosis by saturation and may suppress or activate other macrophage functions" [22]. Consequently, this would have provided inconsistent comparisons between liposometreated and control group animals.

\section{Sciatic Nerve Injury (SNI)}

For all surgical procedures, the toepinch-reflex test was used to determine effectiveness of the anaesthetic prior to surgery using a mixture of ketamine $(100 \mathrm{mg} / \mathrm{kg})$ and xylazine $(100 \mathrm{mg} / \mathrm{kg})$ delivered intraperitoneally. Briefly, a primary longitudinal cut was made on the skin overlaying the femur of the left hind limb. The incision was extended proximally and distally exposing the thigh muscle. Sharp surgical scissors were inserted and opened into the muscle through the first layer to the level at which the sciatic nerve runs. After locating the branching of the sciatic nerve, the nerve was ligated proximal to its trifurcation and cut below the ligation site with fine surgical scissors. The wound was sutured closed using a 6/0 surgical silk suture and animals were placed into individual cages.

\section{Dorsal Column Cut (DCC)}

After laminectomy at T9-T10 and a small incision in the dura mater, the dorsal columns of the spinal cord were crushed with iris scissors inserted at a depth of approximately 1.5-2 mm (marked on the scissors' tip) [23]. A sharp scalpel blade was passed through the wound twice to confirm bilateral DCC [24]. A small piece of gelfoam was temporarily placed over the lesion site to encourage blood clotting and the overlaying muscles were sewn together with a $6 / 0$ surgical suture. The skin was stapled closed and the animals were returned to their cages. After surgery, animals were housed separately and received subcutaneous injections of the analgesic drug buprenorphine $(0.03 \mathrm{mg} / \mathrm{kg})$ for a period of up to 5 days to alleviate postoperative pain. For all spinal cord injured animals manual bladder expression was performed two to three times per day and antibiotics were administered if required [25].

\section{Retrograde Tracer Injection}

To investigate the presence of ascending regenerated fibres in the injured spinal cord (i.e. across the injury epicentre), a somatic retrograde tracer Fast Blue (FB, 5\% in saline, Sigma) was injected into the dorsal column of the proximal stump, 3-4 $\mathrm{mm}$ rostral from the site of spinal cord injury (SCI; injection depth 1-1.1 mm, total volume delivered $0.1 \mu \mathrm{l})$. FB was administered 2 weeks 
after CNS lesion, using a stereotaxic frame and a pulled glass micropipette needle. Note that to alleviate any pain caused by tracer injections into the spinal cord, all animals received injections of buprenorphine $(0.03 \mathrm{mg} /$ $\mathrm{kg}$ ) for up to 3 days after injection.

\section{Anterograde Tracer Injection}

To further examine the presence of ascending CNS fibres regenerating across the $\mathrm{SCI}$ epicentre, the anterograde tracer Biotinylated Dextran Amine (BDA, 10\% in saline, $10000 \mathrm{mw}$, Molecular Probes) was injected into the dorsal column in the lumbar region of the spinal cord [26-28]. BDA was delivered 2 weeks after CNS lesion as described for retrograde tracing (injection depth 1-1.1 $\mathrm{mm}$, total volume delivered $0.1 \mu \mathrm{l}$ ). All care was taken to keep tracer injections within the dorsal column of the spinal cord. Some injected cords were randomly selected to confirm tracer deposition, however, not all injections sites were examined and further damage to the cord at these sites was not investigated in this study.

\section{Liposome Preparation and Administration}

Liposomes were prepared according to previous studies [29-32]. Briefly, $86 \mathrm{mg}$ of egg phosphatidylcholine and 8 $\mathrm{mg}$ of cholesterol were dissolved in $5 \mathrm{ml}$ of chloroform in a round-bottom flask. Chloroform was removed by using a low-vacuum rotary evaporator at $37^{\circ} \mathrm{C}$ to form a thin lipid film around the flask. The lipid was then dispersed with $10 \mathrm{ml}$ sterile phosphate buffered saline (PBS, $0.1 \mathrm{M}, \mathrm{pH}$ 7.4) containing $2.5 \mathrm{gm}$ of clodronate (dichloromethylene-diphosphonate-DMDP, Sigma) and incubated on a gentle stirrer at room temperature (RT, $2 \mathrm{hrs}$ ). After incubation, the suspension was sonicated $(50 \mathrm{~Hz})$ at RT (3 $\mathrm{min})$ and incubated again at RT with no stirring to allow for liposome formation ( $2 \mathrm{hrs}$ ). Liposomes were centrifuged at 10,000 g (15 min) at RT to remove any free clodronate. The remaining pellet was washed twice in sterile PBS at 20,000 g at RT (30 min) and resuspended in $4 \mathrm{ml}$ sterile PBS to be used immediately $[29,30,33]$.

\section{Liposome Administration}

For all test animals, liposomal delivery was administered on three separate occasions, once: i) three days prior to; ii) immediately after; and iii) four days after SNI. The specific timing of liposome administration ensured macrophage depletion commencing 3 days prior to SNI and ending 3 days after DCC. It is known that macrophage cells are depleted within $24 \mathrm{hrs}$ after liposomal treatment and begin to slowly repopulate approximately 5-7 days after last administration of clodronate (personal communication with liposome pioneer Dr Nico van Rooijen, Netherlands). No adverse side effects were observed in any of the liposome-treated animals.

\section{Perfusion and Cryosectioning}

All animals were injected intraperitoneally with 5\% chloral hydrate in distilled water and perfused transcardially with $1 \% \mathrm{NaNO}_{2} /$ phosphate buffer $(\mathrm{PB}, 0.1 \mathrm{M}, \mathrm{pH}$ 7.4) followed by a $4 \%$ paraformaldehyde (PFA)/PB. Perfusions were performed 4 weeks after the CNS lesion with all dissected tissues post-fixed in 4\% PFA and cryoprotected in $30 \%$ sucrose/PB at $4^{\circ} \mathrm{C}$ ( $\left.48 \mathrm{hrs}\right)$. Spleens were cryosectioned at $20 \mu \mathrm{m}$ (cross-sections), DRG at $20 \mu \mathrm{m}$ (coronal sections) and spinal cords at $40 \mu \mathrm{m}$ (longitudinal sections). All specimens were mounted on $2 \%$ gelatine-coated glass slides.

\section{Immunohistochemistry (IHC)}

For IHC, DRG and spinal cord sections were washed in $0.5 \% \mathrm{H}_{2} \mathrm{O}_{2} / 50 \%$ ethanol at RT (30 min) to quench endogenous peroxide activity, rinsed in PBS, followed by three washes in PBS containing 1\% Tween-20 detergent (PBST). These sections were blocked in $20 \%$ normal horse serum (NHS, $2 \mathrm{hrs}$ ) before incubation with primary antibodies at $4^{\circ} \mathrm{C}$ ( $\left.48 \mathrm{hrs}\right)$. The primary antibodies used were: mouseanti-rat cluster differentiation 68 (CD68, macrophage, 1:400, Serotec), rabbit-anti-glial fibrillary acidic protein (GFAP, astrocyte/satellite cells, 1:500, Dako). A combination of the following secondary antibodies for single and/ or double labelling included: sheep-anti-mouse-cy3-IgG (1:500, Jackson), donkey-anti-mouse-cy2-IgG (1:500, Jackson), donkey-anti-rabbit-488-IgG (1:500, Jackson), sheepanti-rabbit-cy3-IgG (1:500, Jackson). The specificity of the observed IHC procedure was validated by omitting the primary antibody and/or by using a non-immune serum instead of the primary antibody [24].

\section{Immunoperoxidase Staining}

BDA injected tissue was treated in $3 \% \mathrm{H}_{2} \mathrm{O}_{2} / 100 \%$ methanol at RT (10 min), rehydrated in PBS and thoroughly washed in PBST. This was followed by incubation with streptavidin-HRP conjugated antibody (1:2000, Vector Laboratories) in PBST at RT $(60 \mathrm{~min})$. After extensive washing with PBST, sections were developed in a solution containing 0.05\% 3'3-diaminobenzidine tetrahydrochloride (DAB, Sigma), $0.06 \% \mathrm{NiSO}_{4}$ and $0.005 \%$ glucose oxidase $[26,34]$.

\section{Relative DRG Somatic Count}

The relative number of retrograde labelled $\mathrm{FB}^{+}$DRG neurons was obtained by serially counting every fourth section from the ipsilateral and contralateral L4 DRG (total of 5 sections/animal, $\mathrm{n}=10$ ). This method of counting allowed for the relative estimation of FB labelled cell bodies and to avoid the possibility of double counting, only those neurons with visible nuclei were counted $[35,36]$. Note that in all instances, $\mathrm{FB}^{+}$neurons were considered regenerated neurons [24]. 


\section{Quantification of Ascending Fibre Regeneration}

The number of BDA-labelled fibres was counted at both stumps of the spinal cord including the lesion epicentre $(0 \mathrm{~mm})$ and $1 \mathrm{~mm}$ rostral and caudal. As we have previously described [37], the axon index was calculated as a percentage of every fourth section (total of $10 \mathrm{sec}-$ tions/animal, $\mathrm{n}=6$ ). Note that no BDA-labelled fibres were found rostrally and/or at the epicentre in liposome-treated animals.

\section{Cellular Quantification Method}

Due to the complexity in identifying individual populations of macrophage cells $\left(\mathrm{CD}^{+} 8^{+}\right)$and astrocytes $\left(\mathrm{GFAP}^{+}\right)$present in the spinal cord after injury, we determined the percentage area fraction of the section occupied by these stained structures $[31,38,39]$. Briefly, using ImageJ (image processing program, NIH version 1.37) $20 \times(697.68 \times 522.72 \mu \mathrm{m})$ magnification images immunostained against the aforementioned antibodies were converted to binary contrast images (black and white). This provided a threshold by subtracting background levels from the immunoreactive stained areas and allowed the determination of the percentage area fraction per image to be collected, tabulated and statistically analysed $[22,38,40,41]$.

\section{Sandwich BDNF ELISA Test}

According to manufacturer's instructions (Millipore. com), serum samples collected at the end of the experimental period were analysed for BDNF concentration level using an ELISA kit. Briefly, samples were incubated overnight at $2-4^{\circ} \mathrm{C}$ in BDNF ELISA plates pre-coated with rabbit anti-human BDNF polyclonal antibody. After incubation, plates were thoroughly washed and incubated at RT (2-3 hrs) with a biotinylated mouse anti-human BDNF monoclonal antibody (1:1000). Plates were then washed and incubated at RT (60 $\mathrm{min}$ ) with a streptavidin-HRP conjugate $(1: 1000)$, washed again, developed with a TMB/E substrate at RT $(15 \mathrm{~min})$. The optical density (OD) was measured at $450 \mathrm{~nm}$ and plotted on a standard curve.

\section{Statistics}

In all graphs, columns represent an averaged mean $(\mathrm{n}=$ 10 and/or as specified per figure) and error bars indicate standard error of mean (+/- S.E.). Comparisons between groups were made using an independent samples t-test. Results were considered significant if $\mathrm{P}<0.05$.

\section{Results}

\section{Effectiveness of Macrophage Depletion}

IHC analysis of spleen sections immunostained to detect macrophage cells $\left(\mathrm{CD} 68^{+}\right)$were used to determine the efficacy of macrophage depletion using clodronate liposomes [22]. Our results revealed a significant difference in macrophage numbers during liposome treatment (1 week after first injection), indicative of successful cellular depletion $(\mathrm{P}<0.0001$; Figure $1 \mathrm{~A}-\mathrm{B} \& 1 \mathrm{I})$. Spleens tested 2 weeks after liposome treatment completion showed early signs of macrophage repopulation evident by $\mathrm{CD} 68^{+}$immunoreactivity in spleens (Figure 1C-D \&1I). However, macrophage numbers had not reached normal levels $(\mathrm{P}<0.05$; Fig. 1I). Comparatively, at the end of the experimental period (day 35) we found a complete repopulation of macrophage cells in spleens of liposome-treated animals, as compared to untreated naïve spleens (Figure 1E-H \&1I).

\section{Retrograde Tracing in DRG}

Quantification of retrograde labelled $\mathrm{FB}^{+}$neurons in the ipsilateral L4 DRG revealed some interesting variations between test and control groups. Consistent with previous observations [7], our results indicated positive retrograde labelling only in the ipsilateral DRG of salinetreated control animals (white arrows, Figure 2A-B), as compared to neurons in the contralateral DRGs (Figure $2 \mathrm{C})$. However, no $\mathrm{FB}^{+}$labelled cell bodies were found in the ipsilateral and contralateral DRGs of liposome-treated animals (red arrows, Figure 2D-F). Statistically, the presence of $\mathrm{FB}^{+}$labelled neurons in the control group as compared to unlabelled DRG neurons in the liposome-treated group indicated a lack of retrograde labelling in the latter group $(\mathrm{P}<0.001$; Figure $2 \mathrm{G})$. These results are indicative of poor attempts at axonal regeneration, possibly attributable to the effects of liposome administration and temporal depletion of macrophage cells.

It seems unlikely that tracer leakage could have affected retrograde labelling of DRG neurons in all of the liposome-treated animals given that this would have resulted in labelled cell bodies in both ipsilateral and contralateral DRG, which was not found (Figure 2G). Note that in all instances, the contralateral DRG was used as the control (uninjured) side. The possibility that regenerated fibres could still be present in the spinal cord of liposome-treated animals was further explored using anterograde tracing.

\section{Anterograde Tracing in Spinal Cord}

Normally in the pre-conditioned lesion model, regenerated ascending afferent fibres extend through the dorsal column of the spinal cord [7]. Here, we assessed this by anterograde BDA labelling of fibres in the dorsal column of the injured spinal cord. Results from saline-treated control animals demonstrated the presence of ascending afferent fibres extending rostrally from the distal stump, across the DCC and into the rostral stump (Figure 3A$\mathrm{H})$. Quantification of BDA-labelled fibres $1 \mathrm{~mm}$ caudal 


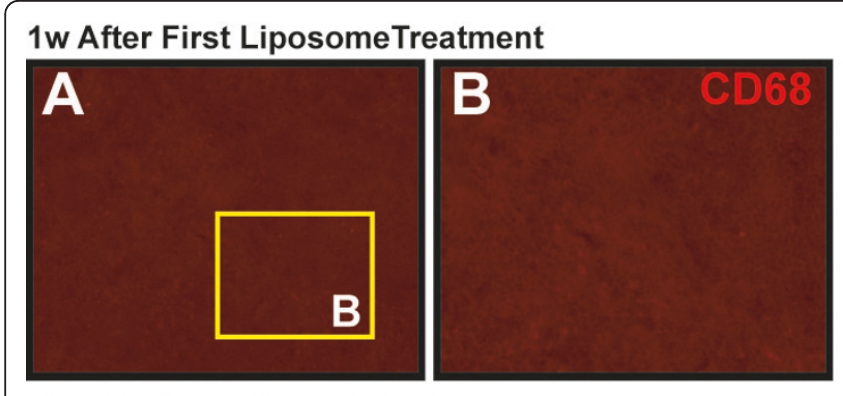

\section{2w Post LiposomeTreatment Completion}
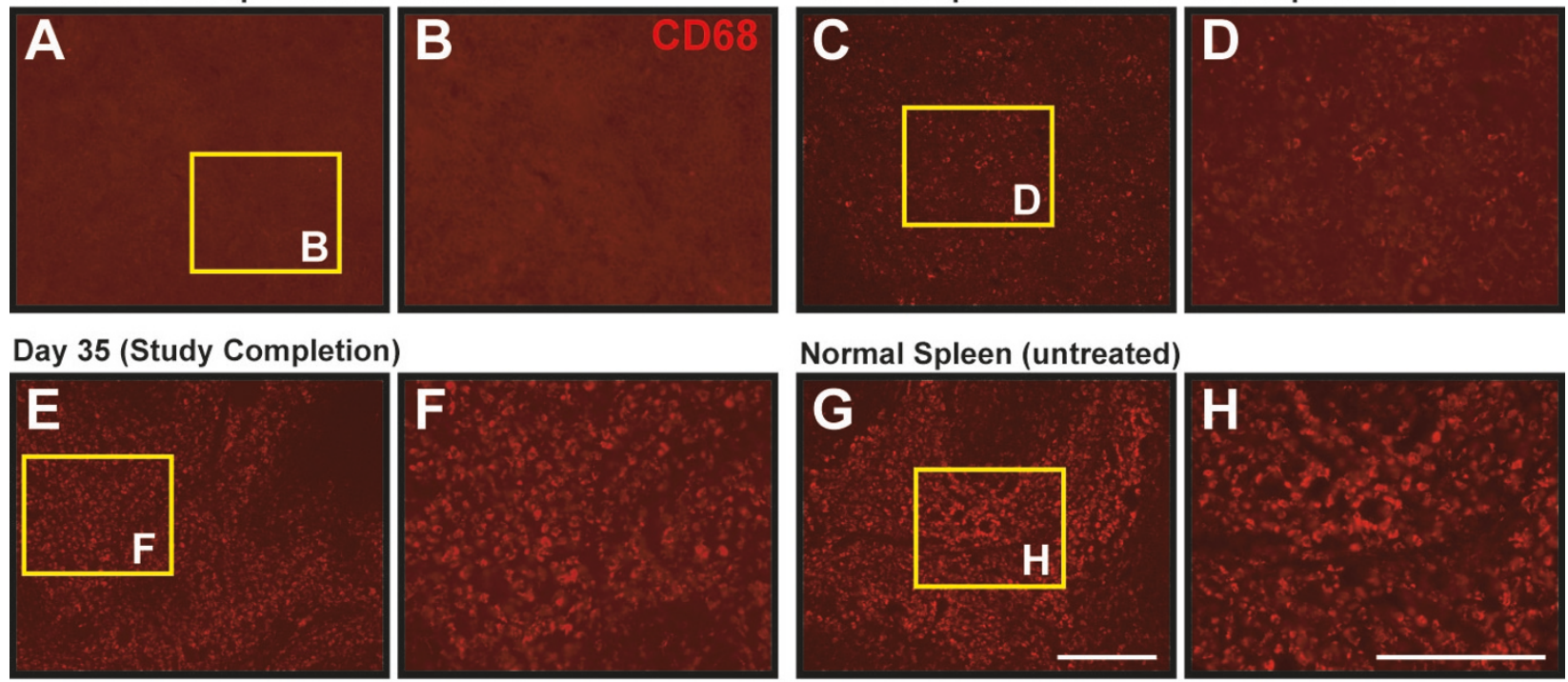

Normal Spleen (untreated)
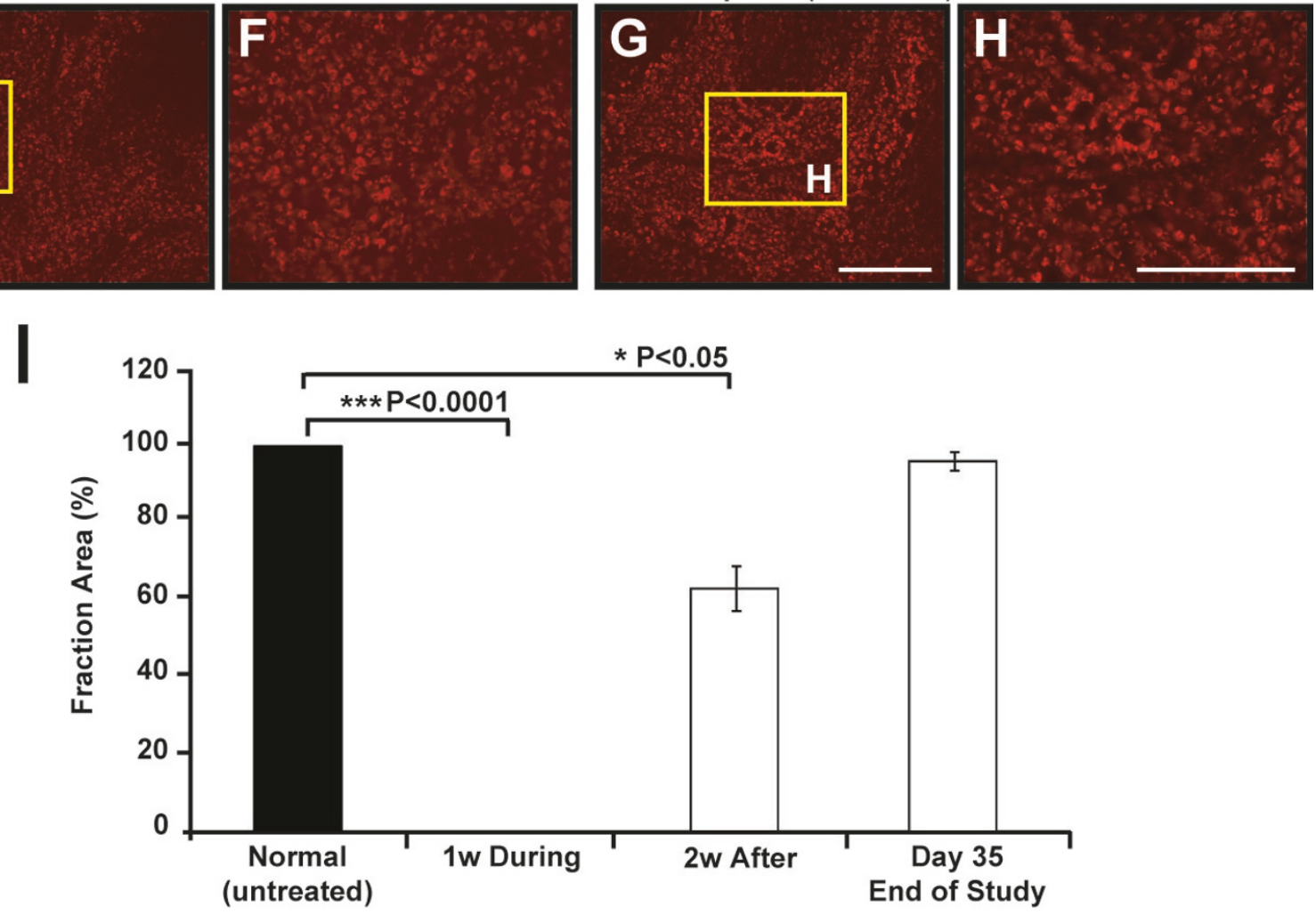

Figure 1 Confirmation of Macrophage Cell Depletion Examined in the Spleens of Liposome-Treated Animals. A-B) 1 week after liposome administration no macrophage cells were present in the spleens of treated animals, as confirmed by the absence of CD68 (macrophage marker) immunoreactivity (***P $<0.0001 ; \mathbf{I})$. C-D) By 2 week after liposome treatment completion, macrophage cells $\left(C D 68^{+}\right)$had already began to repopulate in the spleen of treated animals (*P $<0.05 ; \mathbf{I})$. E-F) At the end of the experimental period (day 35 ) macrophage numbers in the spleen of treated animals had returned to normal levels, as compared to untreated animals (G-H). I) Quantification of immunoreactive CD68 ${ }^{+}$ cells as shown in panels $\mathbf{A}-\mathbf{H}$, demonstrated a significant cellular reduction during liposome administration and during repopulation 2 weeks after treatment completion. No differences in macrophage cells were found at the end of the study. Columns represent an averaged mean (5 sections per animal, $\mathrm{n}=5$ ) and error bars indicate error of mean (+/- S.E.). Scale bars A, C, E, G $500 \mu \mathrm{m}$, enlarged views B, D, F, H $200 \mu \mathrm{m}$.

revealed similar number of ascending fibres close to site of injury in both experimental groups, however, no regenerated fibres were found in liposome-treated animals at the lesion epicentre and $1 \mathrm{~mm}$ rostral $(\mathrm{P}<0.0001$; Figure 3H\&Figure 4). Evidently, in the liposome-treated group labelled fibres in the dorsal column halted at the site of lesion and did not cross the SCI epicentre (Figure 4A-G). Higher magnification images of BDA-labelled fibres in the caudal stump, revealed fibre retraction and collapse close to the site of injury, with no ascending fibres found in the rostral stump (Figure $4 C)$. The lack of regenerated fibres across the SCI epicentre was consistent with the absence of retrograde labelled $\mathrm{FB}^{+}$DRG neurons within this group. This finding confirmed previous assumptions relating to abortive attempts at axonal regeneration and offers tangible explanations for the role of macrophage cells in the preconditioned model of injury. 


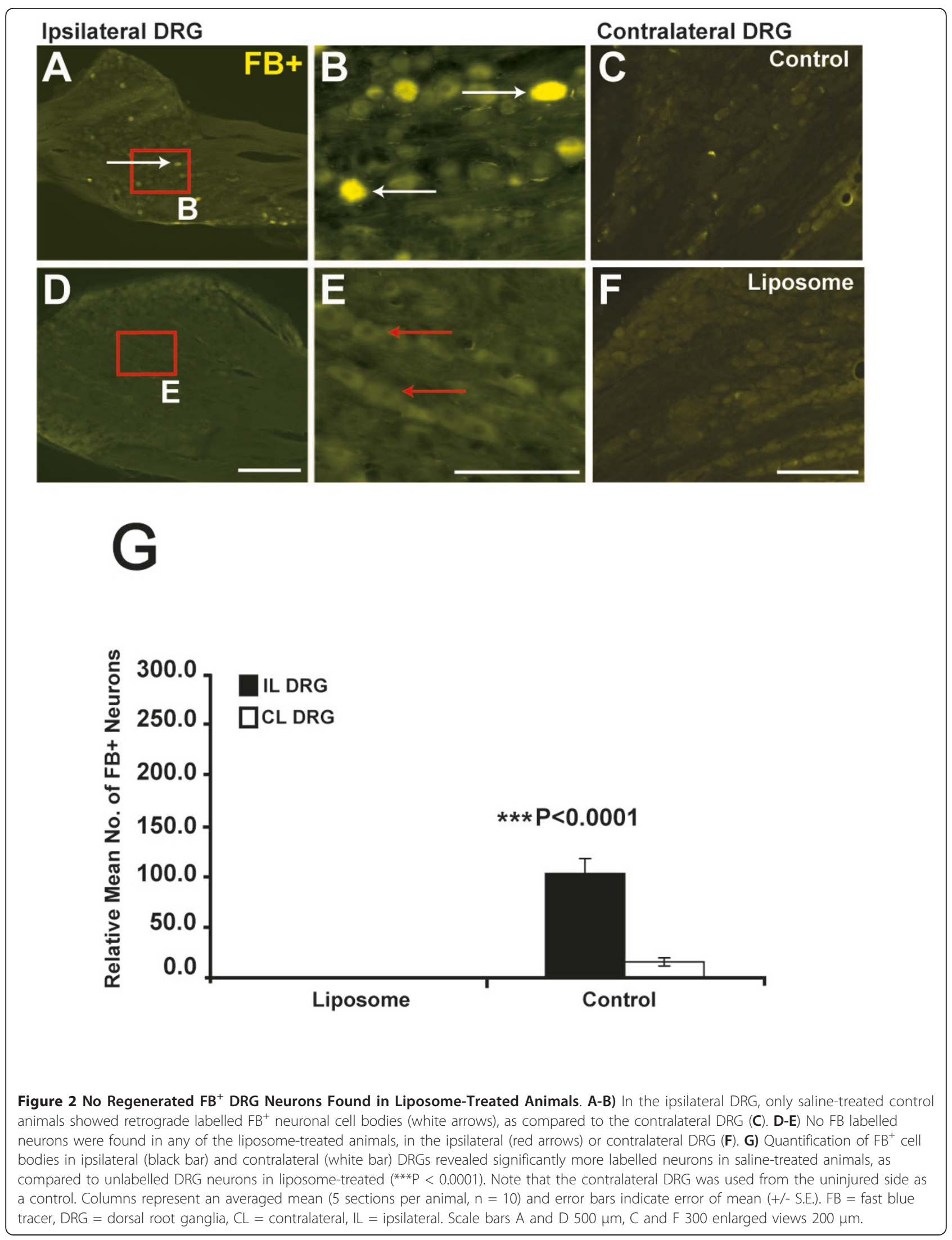




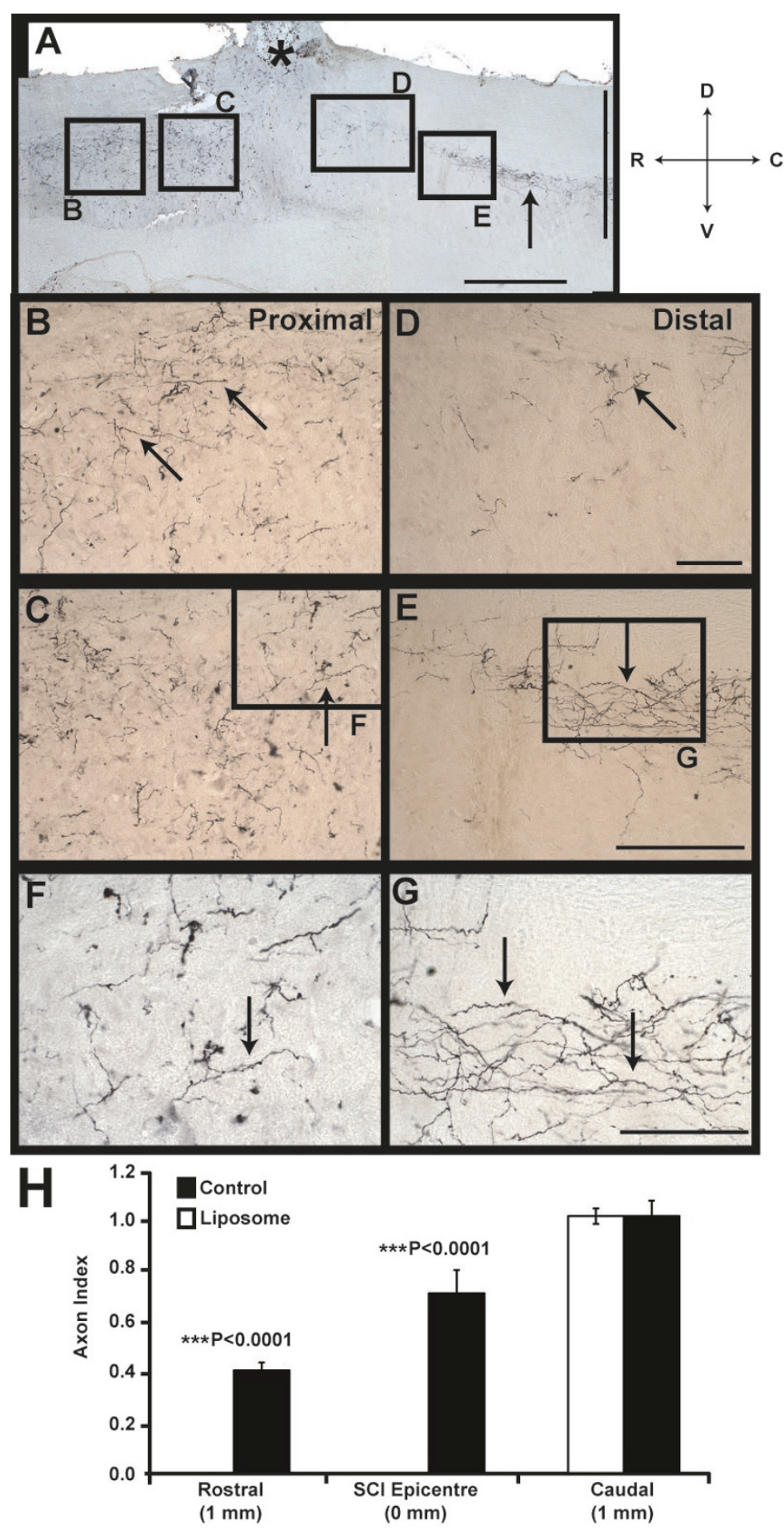

Figure 3 Anterograde BDA-Labelled Fibres in the Spinal Cord of Saline-Treated Animals. A) Montage of SCl epicentre (black asterisk) demonstrating extensive anterograde labelling of ascending fibres in both proximal and distal stumps of the injured spinal cord (black arrows). B-G) Higher magnification images confirming the presence of BDA-labelled fibres extending across both stumps (black arrows), consistent with previous retrograde labelled results described in Figure 2. H) Quantification of percentage of BDA-labelled fibres revealed the presence of regenerated ascending fibres found $1 \mathrm{~mm}$ rostral and at the injury epicentre $(0 \mathrm{~mm})$ in control animals only (***P $<0.0001)$. Similar number of labelled fibres was found $1 \mathrm{~mm}$ caudal in the spinal cord of control and liposome-treated animals. BDA = biotinylated dextran amine, DCC = dorsal column cut. Directional key: $\mathrm{D}=$ dorsal, $\mathrm{V}=$ ventral, $\mathrm{C}=$ caudal, $\mathrm{R}=$ rostral. Scale bars montage $800 \mu \mathrm{m}$ (horizontal bar) and $1 \mathrm{~mm}$ (vertical bar), B -E $200 \mu \mathrm{m}$, F-G enlarged views $100 \mu \mathrm{m}$. 


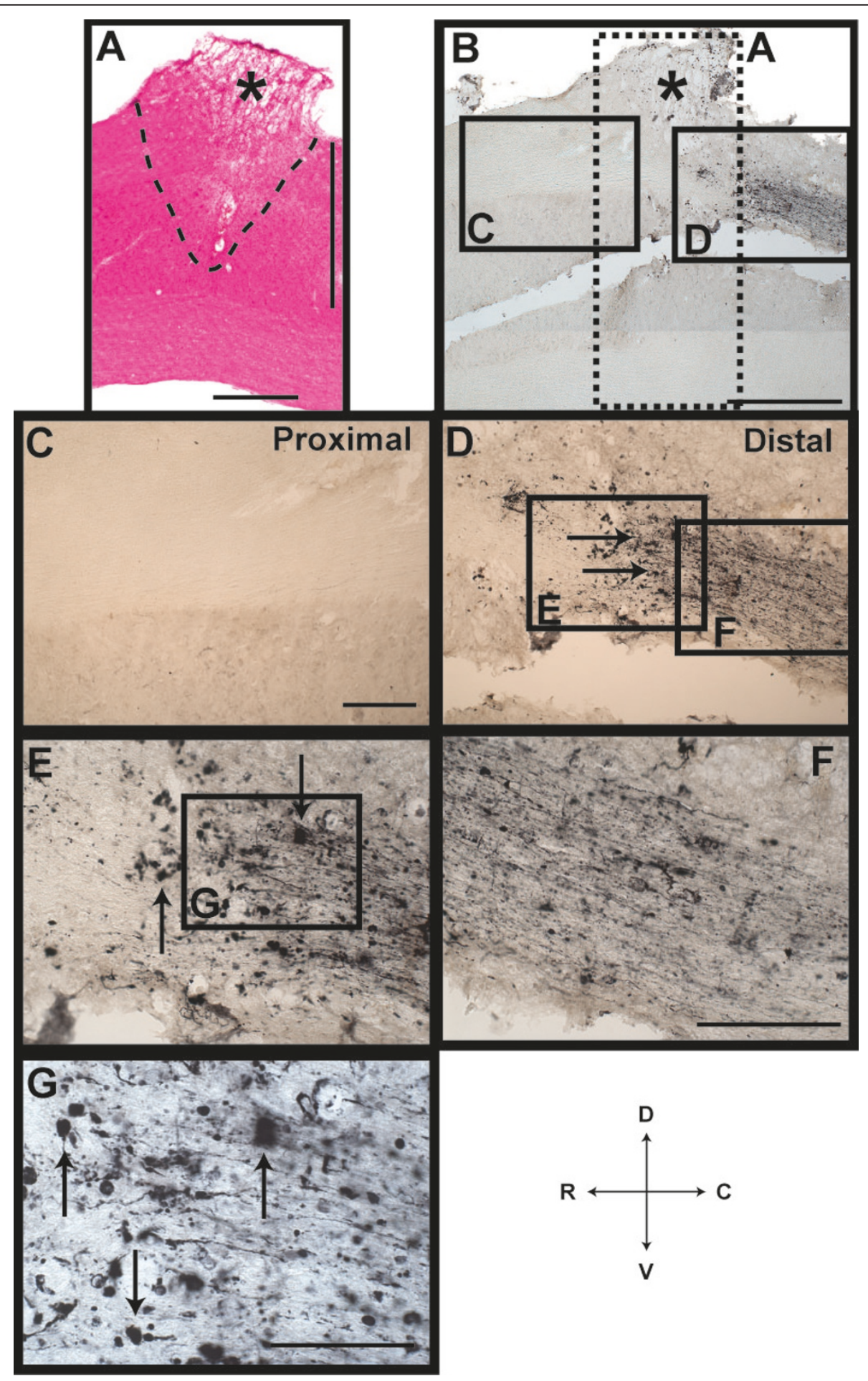

Figure 4 Anterograde BDA-Labelled Fibres in the Spinal Cord of Liposome-Treated Animals. A) Histology-processed section through the $\mathrm{SCl}$ epicentre (black asterisk) revealed extent of lesioning through the dorsal columns (dashed line; shown as insert in panel B). B) Montage of lesion site (black asterisk) demonstrating anterograde BDA-labelled fibres present only in the distal stump of the spinal cord (D), with no visible fibres found in the proximal stump (C). E-G) Closer observation distally revealed extensive neuronal collapse and retraction of BDA-labelled fibres evident by their bulb-like structure (black arrows). BDA = biotinylated dextran amine, $\mathrm{DCC}=$ dorsal column cut. Directional key: $\mathrm{D}=$ dorsal, $\mathrm{V}=$ ventral, $C=$ caudal, $R=$ rostral. Scale bars, A (horizontal bar) $500 \mu \mathrm{m}$ and (vertical bar) $1 \mathrm{~mm}$, montage B $800 \mu \mathrm{m}, \mathrm{C}-\mathrm{E} 200 \mu \mathrm{m}$, F-G enlarged views $100 \mu \mathrm{m}$. 


\section{Macrophage and Astrocyte Quantification}

Macrophage quantification at 1-2 mm both rostral and caudal from the SCI epicentre revealed greater macrophage numbers $\left(\mathrm{CD} 8^{+}\right)$in the saline-treated group, as compared to the liposome-treated group 4 weeks after CNS lesion $(\mathrm{P}<0.05 \& \mathrm{P}<0.01$, respectively; Figure 5A). No statistically significant difference in macrophage numbers between these two groups was found at the SCI epicentre. Similarly, astrocyte quantification $\left(\mathrm{GFAP}^{+}\right) 3 \mathrm{~mm}$ caudal from the lesion site reached similar levels with no statistical differences found between both groups (Figure 5B). Conversely, 3 $\mathrm{mm}$ rostral from the lesion site we found a significantly higher astrocyte expression $\left(\mathrm{GFAP}^{+}\right)$in liposome-treated animals as compared to controls $(\mathrm{P}<0.05)$.

\section{BDNF ELISA Serum Levels}

Calculated serum levels from both treatment groups revealed a significantly higher BDNF concentration level in control animals than in liposome-treated $(\mathrm{P}<0.05$; Figure 6). The fact that immune cells, such as macrophage cells have been reported to support neurite outgrowth by secreting neurotrophic factors such as BDNF $[15,42,43]$, provide a plausible explanation for the lack of regenerated fibres in liposome-treated animals.

\section{Discussion}

\section{Effects of Macrophage Depletion}

The conditioning SNI normally supports the regeneration of CNS afferent fibres of adult DRG neurons [7]. However, we found that the temporal depletion of macrophage cells during the conditioning phase of DRG neurons, consequently abolished the regenerative competence of injured CNS fibres.

Comparisons between injury-matched control and liposome-treated animals revealed a considerable lack of regeneration in the latter group, when compared to retrogradely labelled $\mathrm{FB}^{+}$DRG neurons found in control animals. Concomitantly, anterograde tracing of ascending CNS fibres demonstrated extensive axonal collapse and retraction by the formation of bulb-like structures at the end of axons in liposome-treated animals. In contrast, lengthy BDA-labelled afferent fibres were found in the proximal stump of saline-treated control animals. In addition, astrocyte quantification in liposome-treated animals revealed a higher astrocytic expression level rostral to the spinal cord lesion. This level of astrocyte expression most likely represents the amount of glial scar formation (refer to additional file 1), which may be associated with the observed axonal collapse, as it forms an inhibitory barrier against axonal regrowth [44-46].

Furthermore, macrophage quantification revealed greater numbers in control animals, specifically in regions rostral and caudal to the lesion, as compared to liposome-treated animals. Note that, we do not attribute this variation in macrophage numbers solely to the depletion of these cells, given that: (i) spleens of liposome-treated animals showed macrophage repopulation returned to normal levels; and (ii) liposomal treatment was only temporarily administered, allowing sufficient time for macrophage cells to infiltrate the CNS lesion. This suggests that macrophage presence during the conditioning phase following SNI, is critical for the early activation and guidance of these cells into the CNS compartment [17].

Moreover, BDNF analysis of blood samples demonstrated elevated serum concentration levels in control animals. This difference in endogenous BDNF availability, at least for injured peripheral nerves has been demonstrated to support axonal regeneration and myelination following trauma [47], and is likely to be also contributing to the regeneration of ascending afferent fibres [24]. Certainly, injury to the peripheral DRG branch may be stimulating macrophage cells with a phenotype beneficial for CNS regeneration, as demonstrated using peripheral nerve fragments, reported to enhance phagocytosis and secretion of BDNF $[48,49]$. BDNF is also a nerve trophic factor recently confirmed by our laboratory to be synthesized by macrophages [19] and is a potential mechanistic factor in the successful regeneration of matured CNS neurons after a pre-conditioned lesion (Figure 7).

\section{Macrophage Contribution}

The recruitment number and the phagocytic activity of macrophages has been previously shown to improve the clearance of myelin debris [50], as well as provide important signaling molecules for the improved regeneration of injured axons, dependant on the timing of macrophage activation [51]. This is important given that myelin clearance during Wallerian degeneration by macrophage cells has been reported to be one of the major differences between the PNS and the CNS $[52,53]$. It is also noteworthy that SNI induces circulating macrophage cell infiltration into the uninjured spinal cord, where these cells proliferated and differentiated into microglia cells [17]. This highlights another interesting factor in relation to immune surveillance into the uninjured CNS compartment as it introduces new functions in "bi-directional communication between the CNS and immune system" [54]. Mechanistically, we believe the regenerative trigger characteristic of this model illustrates a complex neuroimmune interaction between the peripheral and central nervous system, regardless of the immune privileged status of the CNS $[55,56]$. 


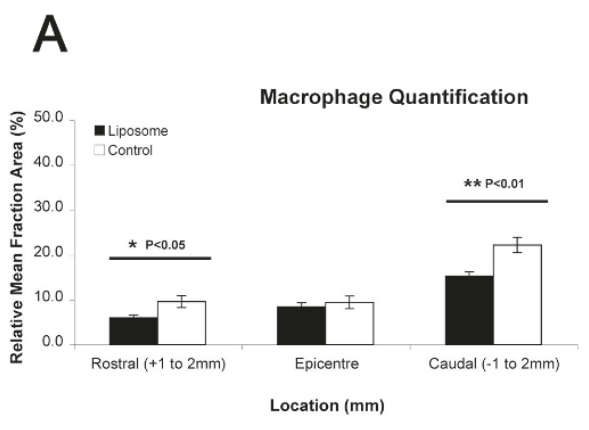

B

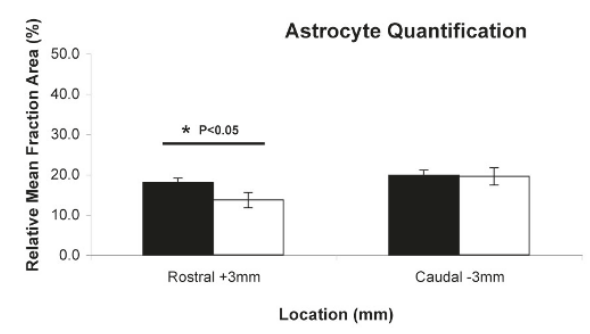

Figure 5 Macrophage and Astrocyte Quantification in the Injured Spinal Cord. A) Statistical analysis of macrophage numbers in the spinal cord of both treatment groups indicated a greater macrophage presence in control animals than in liposome-treated. Specifically, in control animals these numbers were significantly higher 1-2 mm both rostral and caudal from the SCl epicentre, as compared to liposome-treated animals $\left({ }^{*} \mathrm{P}<0.05\right.$, ${ }^{* *} \mathrm{P}<0.01$, respectively), while no differences were found at the $\mathrm{SCl}$ epicentre. B) Astrocyte quantification $3 \mathrm{~mm}$ rostral from the lesion epicentre revealed a greater reduction in astrocyte activation in control animals (*P < 0.05). No differences were observed between groups caudally. Columns represent an averaged mean $(n=10)$ and error bars indicate error of mean (+/- S.E.).

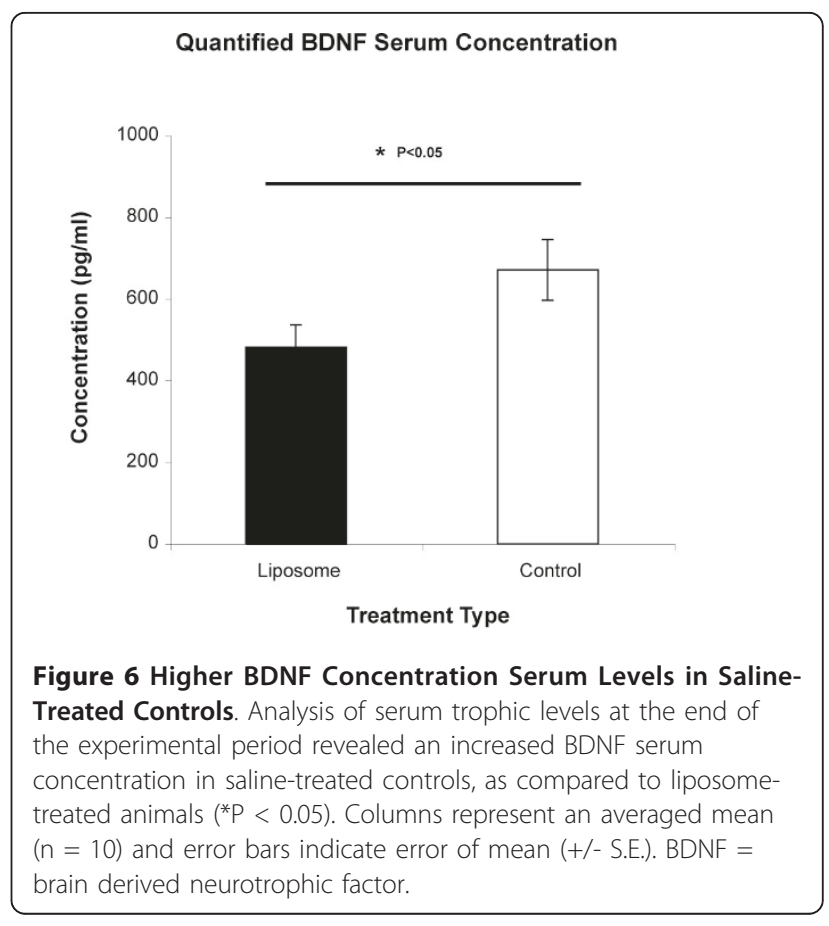

\section{Conclusions}

Despite of the controversy of macrophage cells in CNS repair $[57,58]$, here we ascribe a beneficial role for inflammatory cells in CNS regeneration, given that in vivo macrophage depletion led to astrocyte up-regulation, reduced macrophage infiltration into the CNS and a down-regulation in endogenous BDNF serum concentration. Our data suggest that macrophage activation might be playing a role in the conditioning effect on the regeneration of DRG neurons. These cells are highly

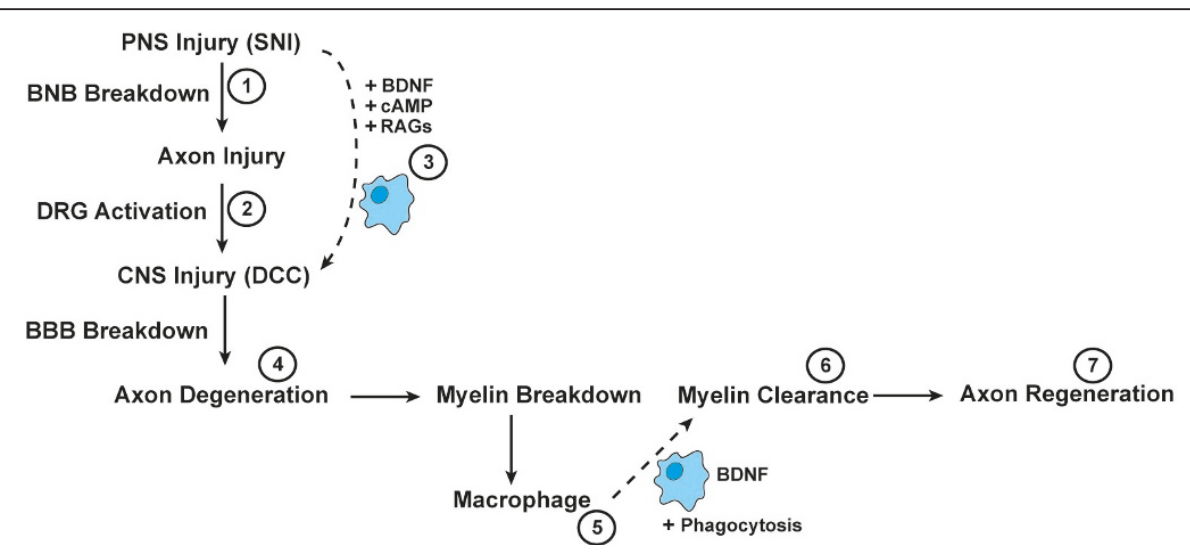

Figure 7 Proposed Mechanistic Model for the Regeneration of Ascending Fibres in Pre-Conditioned DRG Neurons. Regenerative competence of pre-conditioned DRG neurons is described as a multi-faceted cascade of events: (1-2) SNI results in DRG and macrophage activation, upregulation of BDNF, CAMP and regeneration-associated genes (RAGs); all contributing to the beneficial phenotypic 'priming' of macrophage cells. (3) Facilitated entry of primed macrophage cells into the CNS compartment prior to CNS lesion. (4) SCI resulting in axonal injury, axon-myelin breakdown associated with the process of Wallerian degeneration. (5-6) Efficient removal of axonal and myelin debris by BDNF expressing macrophages beneficially activated with enhanced phagocytic capabilities, (7) consequently leading to axonal regeneration of the injured CNS-DRG branch. BNB = blood nerve barrier, RAGs = regeneration-associated genes, BBB = brain-blood barrier. 
attractive for promoting CNS repair, as long as the proregenerative process is not coupled to undesired inflammation.

\section{Additional material}

\section{Additional file 1: Glial Scar Formation at Spinal Cord Injury}

Epicentre. Montage of the spinal cord lesion epicentre demonstrating the extent of glial scar formation after the injury to the dorsal columns. Immunoreactive $\mathrm{GFAP}^{+}$staining identifies the location of lesion epicentre (yellow asterisk), validates the paradigm for astrocyte quantification and delineates the presence of the physical/biochemical barrier against axonal regeneration in the matured CNS (dashed line). Scale bars montage $500 \mu \mathrm{m}$.

\section{Acknowledgements}

This work was supported by Flinders University Research Scholarship to EAAS and a NHMRC grant to XFZ (375110).

\section{Authors' contributions}

EAS carried out all surgical procedure, liposome preparation, $I \mathrm{HC}$, writing of manuscript. ANP carried out statistical analysis, figure preparation and some IHC. MS constructed study design and critical corrections of manuscript. XFZ carried out study design, guidance and drafting of manuscript. All authors read and approved the final manuscript.

\section{Competing interests}

The authors declare that they have no competing interests.

Received: 21 March 2010 Accepted: 20 January 2011

Published: 20 January 2011

\section{References}

1. David S, Aguayo A: Axonal elongation into peripheral nervous system "bridges" after central nervous system injury in adult rats. Science 1981, 214:931-933

2. Caroni P, Schwab M: Two Membrane Protein Fractions from Rat Central Myelin with Inhibitory Properties for Neurite Growth and Fibroblast Spreading. J Cell Biol 1988, 106:1281-1288.

3. Schwab M: Myelin-associated inhibitors of neurite growth and regeneration in the CNS. TINS 1990, 13:452-456.

4. Ramón y Cajal S: Degeneration and Regeneration of the Nervous System London: Oxford University Press; 1928.

5. Richardson P, McGuinness U, Aguayo A: Axons from CNS neurones regenerate into PNS grafts. Nature 1980, 284:264-265.

6. Aguayo A, David S, Bray G: Influences of the glial environment on the elongation of axons after injury: transplantation studies in adult rodents. J Exp Biol 1981, 95:231-240

7. Richardson $\mathrm{P}$, Issa V: Peripheral injury enhances central regeneration of primary sensory neurones. Nature 1984, 309:791-793.

8. Lu X, Richardson P: Inflammation near the nerve cell body enhances axonal regeneration. J Neurosci 1991, 11(4):972-978.

9. Neumann S, Bradke F, Tessier-Lavigne M, Basbaum A: Regeneration of sensory axons within the injured spinal cord induced by intraganglionic cAMP elevation. Neuron 2002, 34:885-893.

10. Qiu J, Cai D, Dai H, McAtee M, Hoffman P, Bregma B, Filbin M: Spinal axon regeneration induced by elevation of cyclic AMP. Neuron 2002, 34:895-903.

11. Cai $D$, Shen $Y$, De Bellard $M$, Tang S, Filbin M: Prior exposure to neurotrophins blocks inhibition of axonal regeneration by MAG and myelin via a cAMP-dependent mechanism. Neuron 1999, 22:89-101.

12. Xu X, Guenard V, Kleitman N, Aebischer P, Bunge M: A combination of BDNF and NT-3 promotes supraspinal axonal regeneration into Schwann cell grafts in adult rat thoracic spinal cord. Exp Neurol 1995, 134:261-272
13. Kobayashi N, Fan D, Giehl K, Bedard A, Wiegand S, Tetzlaff W: BDNF and NT-4/5 prevent atrophy of rat rubrospinal neurons after cervical axotomy, stimulate GAP-43 and Talpha1-tubulin mRNA expression, and promote axonal regeneration. J Neurosci 1997, 17:9583-9595.

14. Mamounas L, Altar C, Blue M, Kaplan D, Tessarollo L, Lyons W: BDNF promotes the regenerative sprouting, but not survival, of injured serotonergic axons in the adult rat brain. J Neurosci 2000, 20(2):771-782.

15. Bouhy D, Malgrange B, Multon S, Poirrier A, Scholtes F, Schoenen J, Franzen R: Delayed GM-CSF treatment stimulates axonal regeneration and functional recovery in paraplegic rats via an increased BDNF expression by endogenous macrophages. FASEB J 2006, 20:E493-E502

16. Dubovy P, Tuckova L, Jancalek R, Svizenska I, Klusakova I: Increased invasion of ED-1 positive macrophages in both ipsi- and contralateral dorsal root ganglia following unilateral nerve injuries. Neurosci Lett 2007, 427:88-93

17. Zhang J, Shi X, Echeverry S, Mogil J, de Koninck Y, Rivest S: Expression of CCR2 in both resident and bone marrow-derived microglia plays a critical role in neuropathic pain. J Neurosci 2007, 27(45):12396-12406.

18. Aguilar Salegio E, Pollard A, Smith M, Zhou X: Sciatic nerve conditioning lesion increases macrophage response but it does not promote the regeneration of injured optic nerves. Brain Res 2010, 1361:12-22.

19. Wong I, Liao H, Bai X, Zaknik A, Zhong J, Guan Y, Li H, Wang Y, Zhou X ProBDNF inhibits infiltration of ED1+ macrophages after spinal cord injury. Brain Behav and Immunity 2010, 24(4):585-597.

20. Van Rooijen N, van Nieuwmegen R: Elimination of phagocytic cells in the spleen after intravenous injection of liposome-encapsulated dichloromethylene disphosphonate. An enzyme-histochemical study. Cell Tissue Res 1984, 238(2):255-258.

21. Van Rooijen $\mathrm{N}$ : The liposome-mediated macrophage 'suicide' technique. $J$ Immunol Meth 1989, 124(1):1-6.

22. Gray M, Palispis W, Popovich P, Van Rooijen N, Gupta R: Macrophage depletion alters the blood-nerve barrier without affecting Schwann cell function after neural injury. J Neurosci Res 2007, 85:766-777.

23. Oiu J, Cafferty W. McMahon S, Thompson S: Conditioning injury-induced spinal axon regeneration requires signal transducer and activator of transcription 3 activation. J Neurosci 2005, 25(7):1645-1653.

24. Song X, Li F, Zhang F, Zhong J, Zhou X: Peripherally-Derived BDNF Promotes Regeneration of Ascending Sensory Neurons after Spinal Cord Injury. PLoS One 2008, 3(3):e1707.

25. Brambilla R, Bracchi-Ricard V, Hu W, Frydel B, Bramwell A, Karmally S, Green J, Bethea J: Inhibition of astroglial nuclear factor kappa-beta reduces inflammation and improves functional recovery after spinal cord injury. J Exp Med 2005, 202:145-156.

26. Novikov L: Labeling of central projections of primary afferents in adult rats: a comparison between biotinylated dextran amine, neurobiotin and Phaseolus vulgaris - leucoagglutinin. J Neurosci Methods 2001, 112:145-154

27. Hughes D, Scott D, Todd A, Riddell J: Lack of evidence for sprouting of Abeta afferent into the superficial laminas of the spinal cord dorsal horn after nerve section. I Neurosci 2003, 23(29):9491-9499.

28. Feng S, Zhou X, Rush R, Ferguson I: Graft of pre-injured sural nerve promotes regeneration of corticospinal tract and functional recovery in rats with chronic spinal cord injury. Brain Res 2008, 1209:40-48.

29. Buiting $A$, Van Rooijen N: Liposome mediated depletion of macrophages: an approach for fundamental studies. J Drug Targetting 1994, 2:357-362.

30. Van Rooijen N, Sanders A: Liposome mediated depletion of macrophages: mechanism of action, preparation of liposomes and applications. $J$ Immunol Meth 1994, 174(1-2):83-93.

31. Popovich P, Guan Z, Wei P, Huitinga I, Van Rooijen N, Stokes B: Depletion of hematogenous macrophages promotes partial hindlimb recovery and neuroanatomical repair after experimental spinal cord injury. Exp Neurol 1999, 158:351-365

32. Van Rooijen N, Kesteren-Hendrikx E: "In vivo" depletion of macrophages by liposome-mediated "suicide". Methods Enzymol 2003, 373:3-16.

33. Claassen E, Van Rooijen N: Preparation and characteristics of dichloromethylene diphosphonate-containing liposomes. J Microencap 1986, 3(2):109-114

34. Shu S, Ju G, Fan L: The glucose oxidase-DAB-nickel method in peroxidase histochemistry of the nervous system. Neurosci Lett 1988, 85:169-171.

35. Li L, Xian C, Zhong J, Zhou X: Effect of Lumbar 5 Ventral Root Transection on Pain Behaviors: A Novel Rat Model for Neuropathic Pain 
without Axotomy of Primary Sensory Neurons. Exp Neurol 2002, 175:23-34.

36. Zhou X, Oldfield B, Livett B: Substance P-containing sensory neurons in the rar dorsal root ganglia innervate the adrenal medulla. J Autonomic Nerv Syst 1991, 33:247-254.

37. Li F, Li L, Song X, Zhong J, Luo X, Xian C, Zhou X: Preconditioning selective ventral root injury promotes plasticity of ascending sensory neurons in the injured spinal cord of adult rats - possible roles of brainderived neurotrophic factor, TrkB and p75 neurotrophin receptor. Eur $J$ Neurosci 2009, 30:1280-1296.

38. Hu P, Bembrick A, Keay K, McLachlan E: Immune cell involvement in dorsal root ganglia and spinal cord after chronic constriction or transection of the rat sciatic nerve. Brain Behav and Immunity 2007, 21:599-616.

39. Piantino J, Burdick J, Goldberg D, Langer R, Benowitz L: An injectable, biodegradable hydrogel for trophic factor delivery enhances axona rewiring and improves performance after spinal cord injury. Exp Neurol 2006, 201:359-367.

40. Maruyama K, Li M, Cursiefen C, Jackson D, Keino H, Tomita M, Van Rooijen N, Takenaka H, D'Amore P, Stein-Streilein J, et al: Inflammationinduced lymphoangiogenesis in the cornea arises from CD11b-positive macrophages. J Clin Invest 2005, 115(9):2363-2372.

41. van Wijngaarden P, Brereton H, Coster D, Williams K: Genetic influences on susceptibility to oxygen-induced retinopathy. Invest Ophthalmol Vis Sci 2007, 48:1761-1766.

42. Deng $Y$, Zhong J, Zhou X: BDNF is involved in sympathetic sprouting in the dorsal root ganglia following peripheral nerve injury in rats. Neurotox Res 2000, 1(4):311-322.

43. Batchelor P, Liberatore G, Wong J, Porrit M, Frerichs F, Donnan G, Howells D: Activated macrophages and microglia induce dopaminergic sprouting in the injured striatum and express brain-derived neurotrophic factor and glial cell line derived neurotrophic factor. $J$ Neurosci 1999, 19:1708-1716.

44. Faulkner J, Herrmann J, Woo M, Tansey K, Doan N, Sofroniew M: Reactive astrocytes protect tissue and preserve function after spinal cord injury. $J$ Neurosci 2004, 24(9):2143-2155.

45. Sofroniew M, Howe C, Mobley W: Nerve growth factor signalling, neuroprotection, and neural repair. Annu Rev Neurosci 2001, 24:1217-1281.

46. Silver J, Miller J: Regeneration beyond the glial scar. Nat Rev Neurosci 2004, 5:146-156.

47. Zhang J, Luo X, Xian C, Liu Z, Zhou X: Endogenous BDNF is required for myelination and regeneration of injured sciatic nerve in rodents. Eur $J$ Neurosci 2000, 12:4171-4180.

48. Zeev-Brann A, Lazarov-Spiegler O, Brenner T, Schwartz M: Differential effects of central and peripheral nerves on macrophages and microglia. Glia 1998, 23:181-190.

49. Lazarov-Spiegler O, Solomon A, Zeev-Brann A, Hirschberg D, Lavie V, Schwartz M: Transplantation of activated macrophages overcomes central nervous system regrowth failure. FASEB J 1996, 10:1296-1302.

50. Vallieres N, Berard J, David S, Lacroix S: Systemic injections of lipopolysaccharide accelerates myelin phagocytosis during Wallerian degeneration in the injured mouse spinal cord. Glia 2006, 53:103-113.

51. Yin Y, Cui Q, Irwin N, Fischer D, Harvey A, Benowitz L: Macrophage-derived factors stimulate optic nerve regeneration. J Neurosci 2003, 23(6):2284-2293.

52. Bruck W, Huitinga I, Dijkstra C: Liposome-mediated monocyte depletion during Wallerian degeneration defines the role of hematogenous phagycytes in myelin removal. J Neurosci Res 1996, 46:477-484

53. Vargas $M$, Barres $B$ : Why is Wallerian degeneration in the CNS so slow? Annu Rev Neurosci 2007, 30:153-179.

54. Quan N, Banks W: Brain-immune communication pathways. Brain Behav and Immunity 2007, 21:727-735.

55. David S, Ousman S: Recruiting the immune response to promote axon regeneration in the injured spinal cord. Neuroscientist 2002, 8:33-41.

56. Pachter J, de Vries H, Fabry Z: The blood-brain barrier and its role in immune privilege in the central nervous system. J Neuropath Exp Neurol 2003, 62(6):593-604.

57. Bethea J: Spinal cord injury-induced inflammation: a dual-edged sword. Prog Brain Res 2000, 128:33-42.

58. Wyss-Coray T, Mucke L: Inflammation in Neurodegenerative Disease-A Double-Edge Sword. Neuron 2002, 35:419-432. doi:10.1186/1471-2202-12-11

Cite this article as: Aguilar Salegio et al: Macrophage presence is essential for the regeneration of ascending afferent fibres following a conditioning sciatic nerve lesion in adult rats. BMC Neuroscience 2011 12:11.

\section{Submit your next manuscript to BioMed Central and take full advantage of:}

- Convenient online submission

- Thorough peer review

- No space constraints or color figure charges

- Immediate publication on acceptance

- Inclusion in PubMed, CAS, Scopus and Google Scholar

- Research which is freely available for redistribution
Ciomed Central 